Daniel Weidner · Sigrid Weigel (Hrsg.)

Benjamin-Studien 1 

Daniel Weidner · Sigrid Weigel (Hrsg.)

Benjamin-Studien 1 
Bibliografische Information der Deutschen Nationalbibliothek

Die Deutsche Nationalbibliothek verzeichnet diese Publikation in der Deutschen Nationalbibliografie; detaillierte bibliografische Daten sind im Internet über http://dnb.d-nb.de abrufbar.

Alle Rechte, auch die des auszugsweisen Nachdrucks, der fotomechanischen Wiedergabe und der Übersetzung, vorbehalten. Dies betrifft auch die Vervielfältigung und Übertragung einzelner Textabschnitte, Zeichnungen oder Bilder durch alle Verfahren wie Speicherung und Übertragung auf Papier, Transparente, Filme, Bänder, Platten und andere Medien, soweit es nicht $\$ \$ 53$ und 54

URG ausdrücklich gestatten.

(C) 2008 Wilhelm Fink Verlag, München

(Wilhelm Fink GmbH \& Co. Verlags-KG, Jühenplatz 1, D-33098 Paderborn)

Internet: www.fink.de

Layout: Marietta Damm, Zentrum für Literatur- und Kulturforschung Berlin Einbandgestaltung: Evelyn Ziegler, München

Printed in Germany

Herstellung: Ferdinand Schöningh GmbH \& Co KG, Paderborn

ISBN 978-3-7705-4637-4 


\section{Von Linearität und Farbe, Zeichen und Mal zum Optisch-Unbewussten im Film. Walter Benjamin in diskursiven Konstellationen mit Gershom Scholem und Siegfried Kracauer}

Der Kunstwissenschaftler Max Imdahl hat in seinen Untersuchungen zur theoretischen Reflexion der Farbe in der französischen Malerei des ausgehenden 19. und des 20. Jahrhunderts die Herausbildung eines nichtbegrifflichen Sehens dargestellt, in dem die Farbinteraktion die Dingdistinktion verzehrt, das "vielfältige und dingüberflutende Wechselspiel der Farben das Auge als Organ belebt« und so die »Dingunterscheidung als Akt rationalen Bewußtseins « ${ }^{1}$ suspendiert. Die grundlegenden Konstellationen einer Debatte um Bildlichkeit und Sehen, Malerei und Graphik, Farbe und Zeichnung in der ersten Hälfte des vorigen Jahrhunderts, die er daraus entwickelt, bezeichnen zugleich jene, in denen auch die frühen, fragmentarischen Überlegungen Walter Benjamins zur Ästhetik, zu Phantasie und Farbe, zu Zeichen und Mal, fortgeführt in Essays wie Aussicht ins Kinderbuch, sich bewegen. Sie korrespondieren mit Konzepten der reinen Farbe und der Bildanschauung eines nicht begrifflichen, nicht vergegenständlichten Sehens, wie sie Baudelaire, Bergson, Proust und Valéry vorgetragen haben, vor allem aber mit der zeitgenössischen Malerei und ihrer Theorie, dem Blauen Reiter und vor allem Wassily Kandinskys Traktat Das Geistige in der Kunst, dessen außerordentliche Auffassung der Farbe Benjamin nur jener der Farbenlehre Goethes zur Seite stellen wollte.

Wenn Benjamin zuerst und vor allem die Farbe vom Kind aus betrachtet, dann macht sich darin ein Spezifisches geltend: neben jener Valérys und Baudelaires sind es Theorie und Literatur, ästhetische und poetologische Theoriebildung der deutschen Frühromantik (immer im Spannungsfeld mit Goethe betrachtet), mit denen seine Auffassung der reinen Farbe korrespondiert, die er um- und fortschreibt. Der Satz aus seinem Essay Aussicht ins Kinderbuch: »reine Farbe ist das Medium der Phantasie, die Wolkenheimat des verspielten Kindes, nicht der strenge Kanon des bauenden Künstlers« (GS IV, 614) formuliert

\footnotetext{
Max Imdahl: Farbe. Kunsttheoretische Reflexionen in Frankreich, München (Fink) 1988, S. 23.
} 
diese Korrespondenzen in größter Dichte. Farbe und Farblosigkeit reflektiert Benjamin zugleich als Medien rezeptiver wie produktiver Aneignung: In-sich-Versenken, ja SichFärben zum einen (das eignet der Farbe in all ihrer Intensität) und Aus-sich-Herausführen (das gilt für Farblosigkeit, Schwarz-Weiß, Umriss und Kontur) erscheinen als Spielarten der Phantasie, die er anthropologisch in einer Körperlektüre des Mimetischen begründet. Den passivisch-rezeptiv-empfänglichen und den aktivisch-hervorbringenden Pol solcher Mimesis reflektiert er in den Begriffen von Entstaltung und Gestaltung - eine Entgegensetzung, die in einigen Fassungen des Kunstwerk-Aufsatzes als Konstellation von Schein (des geschlossenen Werks) und Spiel (offener Charakter ästhetischer Versuchsbildungen) wiederkehrt.

Benjamin stellt früh schon das Farbensehen des Erwachsenen (sein begriffliches, vergegenständlichendes Sehen) dem des kindlichen Menschen gegenüber und nimmt den fundamentalen Umstand, dass Bilder aus visuellen und haptischen Formen bestehen, sogleich und entschieden aus anthropologischer Perspektive in den Blick. Kategorisierungen für den repräsentierenden Gebrauch der Farbe im Sehen des Erwachsenen lauten: Farbe bloß als schichthafter Überzug der Substanz, Farbe hat Eigenschaften, ist bloß abstrahierender Deckmantel individuell einzelner Dinge in Zeit und Raum, konturierende Versachlichung der Dinge. Dagegen gilt für den kindlichen Menschen: Farbe ist Eigenschaft, Farbe wird als nicht fixierend erlebt, sie ist Einzelnes, Besonderes, aber "nicht als tote Sache und eigensinnige Individualität« (GS VI, 110), sondern fluides, volatiles Medium, das alle Verfestigungen des Sehens, alle Bestimmtheit und Konstanz auflöst. Mit diesem Auflösenden aber ist nicht Verschwommenheit, Unschärfe als Gegenwelt statuiert, sondern das Farbensehen ist dadurch in eine andere Perspektive gestellt, dass es an den leiblichen, mimetisch-performativen Vorgang des kindlichen Spiels mit Farben und dem Erzeugen von Farben selber zurückgebunden wird. Das »selbst in die Farbe zerstreut(e)«Wahrnehmen der Kinder (GS VII, 25) bringt im Spiel Ähnlichkeiten zu jener verflüssigenden, fliegenden, durchdringenden »Natur der Farbe» (GS VI, 110) hervor. In ihm erscheint die Farbe vorwiegend, ja überwältigend in haptischer Form, "als Beflügeltes, welches von einer Gestalt zur andern überfliegt.» (Ebd.) Diese »Freude an der Veränderung der Farbe im beweglichen Übergang von Nüancen« (ebd.), am Nicht-Festgestellten, am fließenden, changierenden Spiel der Farben, erneuert sich in abwandelnder Wiederholung, ist nur Spiel und entzieht sich der Determination des Begrifflich-Gegenständlichen. Das Beflügelte der Farbe, welche von einer Gestalt zur anderen überfliegt: diese Erscheinungsweise meint das Bild von der Wolkenheimat der reinen Farbe, meint die Metapher der Wolke, in der sich Farbe als Medium der Phantasie, als unendlich prozessierende Ge- und 


\section{WALTER BENJAMIN UND GERSHOM SCHOLEM}

Entstaltung darstellt. Wolkenwandelbarkeit gilt für die Farbe und perspektivisch ebenso für die Sprache. Reine Farbe kann gewissermaßen als das der Sprache vor- und gleichgeordnete Medium für Benjamins Reflexion der Phantasie angesehen werden. In welchen Überlieferungs-Kontexten dieses Phantasie-Inzitaments seit der Antike und Leonardo sich Benjamin bewegt (eine große Ausstellung hat sie vor einigen Jahren noch einmal in Erinnerung gebracht), macht seine prägnante Wendung vom »Farbensehen als Urphänomen der Phantasieanschauung " sogleich deutlich: der Goethes, aber zugleich und mehr noch, der der Frühromantik. (Ich komme darauf zurück.)

Damit sind, in gedrängter Form, jene Konstellationen von Benjamins Farbdenken umrissen, in die auch eine auf ihre Art eigenartig verquere, nicht eigentlich zu Ende geführte, Debatte mit Scholem über Kubismus und Farbe gehört. Ich nehme sie hier vorwiegend zum Anlass, um den bisher dargelegten Problemaufriss weiter zu verfolgen.

Angestoßen wird Scholems Interesse für den Kubismus durch sein Nachdenken über eine mathematische Theorie der Wahrheit, die er versuchsweise definieren will "als die Lehre von den geistigen Ordnungen der Dinge als mathematischer Beziehungen «. ${ }^{2}$ Sein Besuch der 54. Sturm-Ausstellung im Jahr 1917, in der er zum ersten Mal mit frühen kubistischen Bildern konfrontiert wird, scheint diese Spekulationen auf unerwartete Weise in der Sphäre der bildenden Kunst zu bestätigen. Diesem Eindruck steht nur die überwältigende Wirkung entgegen, die die leuchtende Farbigkeit der Bilder Marc Chagalls auf ihn macht. Sie erscheint als »reine Metaphysik«, doch auch die »Teilung des Raumes" auf diesen Bildern »ist ungeheuer «. ${ }^{3}$ Scholem hatte Kenntnis von Benjamins ersten philosophisch-dialogischen Versuchen über die Farbe, hatte kleine improvisierte Vorträge über die Farbigkeit illustrierter Kinderbücher miterlebt, und so widmet er sich sogleich dem Problem, das die Entwicklung des Kubismus (wenn man so will: vom analytischen zum synthetischen) entscheidend mitbestimmt hat: eben dem Problem der Farbe und der Farblosigkeit.

Er meint einen unaufhebbaren Widerspruch zwischen der kubistischen Formensprache und der glühenden Farbigkeit auf einigen frühen Gemälden Chagalls zu erkennen. Während er Chagalls Farben attestiert, sie seien reine Metaphysik, werden dessen kubistische Versuche prinzipiell verworfen, weil dieser "vollkommen un-mathematische Mensch « ${ }^{4}$ seinen "reine(n) Weg ${ }^{5}$ nur in der symbolischen Erkenntnis der Welt in Farben und unter

2 Gershom Scholem: Tagebücher nebst Aufsätzen und Entwürfen bis 1923, hg. v. Karlfried Gründer u.a., 2. Halbbd. 1917-1923, Frankfurt a.M. (Jüdischer Verlag) 2000, S. 26.

Ebd., S. 30.

Scholem: Tagebücher (Anm. 2), S. 33.

Ebd., S. 31. 


\section{HEINZ BRÜGGEMANN}

Verdammung der Linie gehen könne. Mit seinem Versuch, »das Wesen des Raumes durch Zerlegung auszudrücken« und »durch die Linie mitzuteilen«, steht der Kubismus in der "nächste(n) Nähe zur Mathematik", was Scholem dazu führt, die vollkommene Farblosigkeit (und Formlosigkeit) des "genial« kubistischen Bildes zu fordern: »Farbe darf in dieser Welt, in der der Raum in metaphysische Zellen zerlegt wird, kein Element sein«, sie gehört nicht hierher, "weil sie a-mathematisch ist - der Kubismus habe die Form überwunden, er werde »die Farbe überwinden " und »eine wabre Erkenntnis sein «, ${ }^{6}$ ja er ist »der künstlerische Ausdruck der mathematischen Theorie der Wahrheit«. ${ }^{7}$ Scholem hat bei diesen so rigoros vorgetragenen Bemerkungen offenbar vor allem den sogenannten analytischen Kubismus der Anfänge vor Augen - Picassos Dame mit Fächer von 1909 wird genannt und als Kitsch verworfen, weil die Dame und der Fächer zu sehen seien; dagegen wird Frau mit Violine gepriesen. Mit seinem monochromen Grisailleton, allenfalls seiner Zweifarbigkeit, seiner Konzentration auf die Form, auf Umriss und Volumen als dem Primären (keineswegs auf Formlosigkeit, wie Scholem meint, dem so etwas wie der reine, farblos gemalte Begriff vorschwebt) misstraute er in der Tat den Farben als sekundären Eigenschaften, flüchtigen und zufälligen Elementen. In Scholems Argumentation gehen, ohne dass er das kenntlich machen müsste, da der Bezug den debattierenden Freunden ohnehin deutlich war, Motive des Neukantianismus ein. Mehr noch: am Ende scheint ihm die Kunst des Judentums überhaupt "auf der symbolischen Zerlegung des Raums zu beruhen «, im beinahe kubistisch wirkenden Lebensbaum, in der Menorah etc. offenbart sich die »tiefe Beziehung des Judentums zur Mathematik", die jüdische Kunst »beruht nicht auf dem Bildnis, sondern auf der starren großen Linie, ja das jüdische Bild eines Menschen muß kubistisch sein. ${ }^{8}$ Von dem so groß gedachten mathematischen Symbol muss verlangt werden, so Scholem kategorisch, dass seine „Größe [...] auch die Farbe mit-erkennt. Farbe darf in dieser Welt, in der der Raum in metaphysische Zellen zerlegt wird, kein Element sein, sonst ist die ganze, nun dualistische Anschauung a priori verbürgerlicht. « ${ }^{9}$

Scholem teilt die Prämissen Kants, der Raumeigenschaften und Farbeigenschaften nicht denselben Status zugesteht - Farben sind im Gegensatz zur transzendentalen Ideali-

\footnotetext{
Ebd., S. 32 .

Ebd., S. 33.

Ebd., S. 33 u. 34. Es dürfte das erste Mal sein, dass eine bildnerische Formensprache der klassischen Moderne ganz für eine monotheistische Religion in Anspruch genommen wird. Die zionistisch motivierte Zuschreibung der modernen, formalen Mathematik an die Juden, die dem zugrunde liegt, teilt Scholem zu diesem Zeitpunkt allenfalls mit Theodor Lessing, der von der mathematischen Begabung der Juden gesprochen hatte. Vgl. dazu den instruktiven, solche Mythologeme auflösenden Aufsatz von Herbert Mertens: "Jüdische Mathematik?«, in: Daniel Tyradellis/Michael S. Friedlander (Hg.): $10+5$ = Gott. Die Macht der Zeichen, Köln (DuMont-Literatur-und-Kunst-Verlag) 2004, S. 183-197.

9 Ebd., S. 32.
} 
tät der raum-zeitlichen Eigenschaften keine notwendigen Bedingungen der Körper, sondern bloß zufällige Wirkungen, kausale Affektionen durch Licht, bloße Modifikationen des (empirischen) Subjekts. ${ }^{10}$

Es ist diese kantianische und von der Mathematik her begründete apriorische Wertigkeit des Raumes, die Scholems enthusiastische Haltung gegenüber dem frühen analytischen Kubismus bestimmt. Er hat Farbe darauf reduziert, die Formen der Volumina zu bestimmen, den Gegenstand von verschiedenen Seiten zu zeigen, von oben und von unten - und damit gleichsam auch die Zeit selber unter die Formen seiner künstlerischen Anschauung a priori mitaufzunehmen -, ihn aufzubrechen, um seinen Schnitt oder sein Inneres zu veranschaulichen etc. All das hat dazu geführt, so hat es Daniel Henry Kahnweiler in seinem Buch über Juan Gris dargestellt, »im monochromen Grisailleton der Bilder phantastische Konstruktionen « ${ }^{11}$ aufzutürmen. Mit dieser Monochromie, allenfalls Zweifarbigkeit, kommt für Scholem mehr als jener Pol der bisher aufgespannten Konstellationen ins Spiel, den wir Umriss und Kontur, ja Gestalt genannt haben, nämlich die Schrift. Er hält im Tagebuch fest: "Das Wesen des Kubismus geistig gesehen ist: das Wesen des Raumes durch Zerlegung auszudrücken. Ungeheure Symbole (der Halbkreis, die Senkrechte und die Horizontalen, [...] die alle von höchster metaphysischer symbolischer Bedeutung sind), sind die Funktionen ihrer Welterkenntnis. Dies rückt sie in die nächste Nähe zur Mathematik (wenigstens zu meiner Auffassung von Mathematik). « ${ }^{12}$ Weniger aus der Mathematik freilich als aus dem Veranschaulichungsmaterial der Wahrnehmungspsychologie um 1900 hat man so etwas wie das raumstruktive Vokabular des Kubismus (Machs Visitenkarte, Eggers Becher, Parallelepided, Abgestumpfte Pyramide, Schröders Treppe etc.) zusammengesetzt. ${ }^{13}$ Es ist der Begriffsund Schrift-Charakter dieser Bilder, ihre gleichsam farblose Begriffsschrift, die Scholem zur kulturellen, ja theologischen Kodierung dieser kubistischen Formensprache einlädt - i.d. Fall Kubismus als mathematisch-raumzerlegende Bildsprache des jüdischen Monotheismus.

Benjamin hat dem Primat der mathematischen Raumkonstruktion und dem monochromen Zeichencharakter in Scholems Aneignung entschieden, wenn auch »rein polemisch" widersprochen, (...) »ohne noch eine selbständige Einordnung des Kubismus zu versuchen $«$.

10 Vgl. dazu den Aufsatz von Johannes Haag: "Kant und die Farben«, in: Jakob Steinbrenner/Stefan Glasauer (Hg.): Farben. Betrachtungen aus Philosophie und Naturwissenschaften, Frankfurt a.M. (Suhrkamp) 2007, S. 102-125.

11 Daniel-Henry Kahnweiler: Juan Gris - Leben und Werk, Stuttgart (Hatje) 1968, S. 118.

12 Scholem: Tagebücher (Anm. 2), S. 31.

13 Vgl. den immer noch einschlägigen Aufsatz von Marianne L. Teuber: "Formvorstellung und Kubismus oder Pablo Picasso und William James«, in: Kubismus. Künstler, Themen, Werke 1907-1920, Köln 1982 (= Ausstellungskatalog), S. 9ff., bes. S. 27. 


\section{HEINZ BRÜGGEMANN}

In Scholems Bestimmung des Kubismus, »das Wesen des Raumes der die Welt ist durch Zerlegung mitzuteilen«, scheint ihm »ein Irrtum bezüglich des Verhältnisses der Malerei zu ihrem sinnlichen Gegenstande vorzuliegen« (GB I, 395). Zwar könne man »in der analytischen Geometrie die Gleichung eines zwei- oder dreidimensionalen Gebildes im Raume geben ohne durch sie aus der Analyse des Raumes herauszutreten; nicht aber in der Malerei Dame mit Fächer (z.B.) malen, um damit das Wesen des Raumes durch Zerlegung mitzuteilen. Vielmehr muss die Mitteilung unter allen Umständen durchaus »Dame mit Fächer« betreffen« (ebd.). Benjamins Einwand scheint überzeugend, wenn man bedenkt, dass die beschworene unerhörte Freiheit, die neue Sprache der kubistischen Malerei, zunächst einmal darin besteht, "nicht mehr an das mehr oder weniger "naturähnliche» optische Bild gebunden zu sein, das ein einziger Standpunkt von einem Gegenstande gewährt «. ${ }^{14}$ Der Gegenstand entsteht sozusagen aus dem Raumkonstruktiven, er beherrscht es nicht mehr. Die dritte Dimension wird nicht nachgeahmt, sondern bildgemäß gestaltet, durch die Verformung des dreidimensionalen Bewegungserlebnisses in zweidimensionale Gestalt. Die starke Selbständigkeit der kubistischen Konzeption mit ihrer Dominanz des Raumkonstruktiven, der stereometrischen Zeichnung in der Fläche, dem Nebeneinander flächenhafter, doch verschiedener Sichten, hat dazu geführt - wie Kahnweiler schon in seiner ersten, unter dem Pseudonym Daniel Henry publizierten Schrift über den Kubismus feststellt -, dass die ersten Betrachter in den Gemälden geometrische Formen ısahen`. Das ist der Zustand, den Scholem in der Diskussion von 1917 gewissermaßen logokratisch verabsolutiert. Kahnweiler zeigt dagegen, dass die vom Betrachter verlangte Leistung der Assimilation an diese neue Sprache der Malerei dadurch beschleunigt werde, dass kubistische Bilder stets mit beschreibenden Titeln versehen werden, wie 'Flasche und Glas`, sSpielkarten und Würfel etc., weil dadurch der Praeperception genannte Zustand und die mit dem Titel zusammenhängenden Erinnerungsbilder sich leichter einstellen. Sodann argumentiert er ganz ähnlich wie Benjamin drei Jahre zuvor: dieser "geometrische Eindruck beim Beschauer" sei "unberechtigt, da die vom Maler gewünschte Sehvorstellung nicht etwa in geometrischen Formen besteht, sondern in der Darstellung der wiedergegebenen Gegenstände. ${ }^{15}$ Nicht darum könne es sich handeln, `Dame mit Fächer` zu malen, um damit das Wesen des Raumes durch Zerlegung mitzuteilen, sondern die Mitteilung müsse `Dame mit Fächer betreffen - so die Zuspitzung bei Benjamin.

Im Winter 1926/27 hatte er im Moskauer Puschkin-Museum Gelegenheit (oder: hätte er Gelegenheit gehabt), Pablo Picassos Dame mit Fächer von 1909 in der Samm-

14 Daniel Henry: Der Weg zum Kubismus, München (Delphin-Verlag) o.J., S. 34.

15 Ebd., S. 35. 
lung S.I. Schtschukin zu sehen - ein ganz frühes kubistisches Bild, das seiner Argumentation bis zu einem gewissen Grade entspricht. Wenn er auch die Voraussetzung, die Kahnweiler in kantianischer Manier nachträgt, nicht explizit macht: dass Kubus, Sphäre, Zylinder fest im Menschen verankerte Formen der Anschauung seien, Sehkategorien, primäre Qualitäten, und es für uns ohne »unsere Kenntnis a priori dieser Formen [...] kein Sehen, keine Körperwelt gäbe. «16

Nicht mitvollziehen will Benjamin Scholems Versuch, den Kubismus für eine neokantianisch inspirierte Verwerfung des Bilderdienstes, der schillernden Farbenvielfalt des Natürlichen und seiner rauschreichen und imaginativen Welt zu funktionalisieren. Sein Weg führt in eine Ausweitung des Begriffs der Erfahrung über die Grenzen Kants, ins Mimetische, Rauschreiche und Imaginative, in die Farbe und die Sprache.

In seinem Text Über die Malerei oder Zeichen und Mal, der sich auf diesen Briefwechsel bezieht (GS II, 603-607, Kommentar GS II, 1412-1415; GB I, 393ff.) - »eigentlich kein Aufsatz, sondern zu einem solchen erst der Entwurf" (GB I, 393) - versucht Benjamin die Auseinandersetzung prinzipiell zu wenden und anthropologisch tiefer zu legen. Er resümiert zunächst, Scholems Darstellung aufnehmend, das Problem des Kubismus liege "von einer Seite her gesehen in der Möglichkeit einer, nicht notwendig farblosen, aber radikal unfarbigen (...) Malerei in der lineare Gebilde das Bild beherrschen - ohne dass der Kubismus aufhörte Malerei zu sein und zur Graphik würde.« (GB I, 394)

Benjamin setzt sodann - seinem Denken in Polaritäten folgend - gegen die Hypertrophie der Verfügbarkeit im begrifflichen Sehen eine Hypertrophie des Unverfügbaren, mit der psychophysisch angelegten anthropologischen Konstruktion eines sog. »absoluten Mals«im Sinne von Erröten, Aussatz, Muttermal. Dieses absolute Mal, eine psychologisch oder physiologisch bewirkte, unmittelbare Hervorbringung von Farben aus Natur (Blut, Hautverfärbung etc.) bringt er mit der Malerei in Verbindung, indem er das Verhältnis von Farbe und Raum einführt: Malerei sei ein Mal im engeren Sinne, da sie »weder Untergrund noch graphische Linie kennt«(GS II, 606). Ausschließender könnten die Extreme dieser Polarität nicht gegeneinander geführt werden: erscheint das »Mal« doch wie eine material aufgefasste farbige Entität, eine Matrix, in die die Komposition als eine Art höhere Macht eintritt und die Beziehung auf das, "wonach das Bild benannt wird", mithin »auf das dem Male Transzendente« (GS II, 607) herstellt. Ist die Argumentation bis hierher deutlich gegen den bestimmenden, prägenden (machtvollen) Auftritt des Zeichens (der Komposition) in Gestalt des graphischen Gebildes gerichtet, erfährt sie nun eine Wende

16 Ebd., S. 40. 


\section{HEINZ BRÜGGEMANN}

mit der Einführung eines Gemeinsamen (einer Ähnlichkeit, Verwandtschaft) von Komposition und Mal. Diese Verwandtschaft aber besteht darin, dass beide sprachlichen Wesens seien, was Benjamin zu einer schönen und für sein Farbdenken entscheidenden Wendung führt: "Diese Macht [scil. der Benennung, der Namengebung H.B.] ist das sprachliche Wort, das sich im Medium der malerischen Sprache, unsichtbar als ein solches, nur in der Komposition sich offenbarend, niederläßt.« (GS II, 607). Das Bild werde nach der Komposition benannt, somit verstehe es sich von selbst - direkt gegen Scholem gewandt, der wie erinnerlich die Farbe ganz aus dem kubistischen Bild als reiner Begriffsschrift verbannt sehen wollte - , dass »Mal und Komposition Elemente jedes Bildes sind, welches auf Benennbarkeit Anspruch macht." Die Betonung liegt auf »und - ein Bild, das dies nicht täte, würde "aufhören ein solches zu sein« und »in das Medium des Mals überhaupt« eintreten - "wovon wir uns aber gar keine Vorstellung machen können. (Ebd.)

Wenn Benjamin die Komposition als ein unsichtbares sprachliches Wort einführt, das im Medium der verwandten malerischen Sprache und im dialogischen Bezug mit ihm die Benennung des Bildes hervorbringt, dann ließe sich die Differenz zu Scholem und in gewisser Weise auch zum Kubismus so beschreiben: Im Kubismus tritt das benennende Wort wie ein Zeichen zum Bild hinzu, um den assimilierenden, synthetisierenden Sehprozess des Betrachters zu initiieren und ihm eine Richtung zu geben. Benjamin löst das darin immer noch enthaltene Moment des aufprägenden Zeichens, der Signifikation, ab durch einen Dialog malerischer und kompositioneller Sprachgebärden, aus dem das benennende Wort im Idealfall wie von selber heraustritt. Man könnte hinzufügen, dass da, wo sich dieses nicht ereignet, die frühen abstrakten Bilder (etwa Kandinskys) keinen Gegenstand benannt haben, sondern das malerische Geschehen, das Verfahren selber zum Bildtitel wählten, zumeist allerdings (eine Dimension, die Benjamin außer Acht lässt) im Medium der Farbe-Ton-Beziehung, eben »Komposition«, oder dem Charakter des Gesprächs zwischen der malerischen Sprache und dem Wort der Komposition folgend: Improvisation. Der Eindruck drängt sich auf, dass Benjamin weniger kubistische als Bilder von Kandinsky oder Klee vor Augen gestanden haben - vor allem bei letzterem gibt es immer wieder ein unabweisbar evidentes So-und-nicht-anders als Heraustreten des benennenden Titels aus dem Bild, aus Mal und Komposition (etwa: Rosenwind, Feuer-Geist, es dämmert und viele andere).

Folgen Benjamins frühe Überlegungen und Dialoge (seit 1914/15) zumeist dem materialen kindlichen Spiel mit Farben, so kommen sie doch zugleich auch den Erscheinungsformen und der Bildphänomenalität der vom Gegenstand und seinem Ort emanzipierten Farben erstaunlich nahe, wie sie in der zeitgenössischen Malerei der von ihm 
hochgeschätzten Maler August Macke, Paul Klee (auch Marc Chagall) und vor allem Wassily Kandinsky zu Tage treten. Vergleichsweise seien neben Benjamins Bestimmungen der "Farbe der Phantasie« selber: sie sei ohne Übergänge und spiele doch in unzähligen Nüancen, sie verwische die Dinge in der Färbung ihrer Kontur, sei reine Eigenschaft von keiner Substanz, »eine farbige Ausfüllung des einen Unendlichen durch Phantasie « (GS VII, 25), entsprechende Ausführungen von Wassily Kandinsky aus dem Geistigen in der Kunst gestellt (dazu gehört ein Aquarell, das in Fünffarbendruck im ersten Heft des 11. Jahrgangs der Zeitschrift Der Sturm, 1920, reproduziert war ${ }^{17}$ - das Heft befindet sich in dem kürzlich in Moskau gefundenen Nachlass-Teil Benjamins). Bei Kandinsky heißt es über das »unendlich große Arsenal der Ausdrucksmöglichkeiten«, die die vom Gegenstand emanzipierte Farbe gerade zu erschließen beginnt:

»Erlaubte«, »unerlaubte« Zusammenstellungen, der Zusammenstoß der verschiedenen Farben, das Übertönen einer durch die andere, vieler durch eine, das Herausklingen einer aus der anderen, das Präzisieren des farbigen Fleckes, das Auflösen ein- und vielseitiger, das Zurückhalten des fließenden Farbenfleckes durch zeichnerische Grenze, das Übersprudeln dieses Fleckes über diese Grenzen, das Ineinanderfließen, das scharfe Abtrennen usw. usw. eröffnen eine sich in unerreichbare Fernen verlierende Reihe der reinmalerischen (= farbigen) Möglichkeiten. ${ }^{18}$ Vielleicht verstellen sich Benjamins fragmentarische Reflexionen durch die Analogie der im Artefakt verwandten Farbe als einem materialen Medium mit dem Unverfügbaren psycho-physisch-bedingter Färbung mehr als sie erhellen. Denn für die abschließende Wendung, dass das Bild, das auf Benennbarkeit keinen Anspruch machte, aufhören würde "ein solches zu sein und nun freilich mit in das Medium des Mals überhaupt eintreten" würde, "wovon wir uns aber gar keine Vorstellung machen können« (GS II, 607). Man mag dabei an Verfahren denken, wie sie Max Ernsts Frottagen, Jackson Pollock mit dem dripping, der Tachismus, die Nouveaux Réalistes (etwa mit den Plakatabrissen eines Raymond Hains) praktiziert haben. Schon in der Entwicklung des Kubismus selber, auch des Dadaismus, ist ein unmittelbarer Umgang mit dem Materialen nicht allein der Farbe, sondern mit dem Stofflichen aus Natur und Kultur beschlossen, der deren Unverfügbares in hohem Maße gegen das Zeichen ausspielt, sodass man sich davon im Oktober 1917 vielleicht noch keine Vorstellung machen konnte (ich komme darauf zurück).

17 Eine Abbildung findet sich in meinem Buch: Walter Benjamin über Spiel, Farbe und Phantasie, Würzburg (Königshausen \& Neumann) 2007, Abb. 15, vor S. 113.

18 Wassily Kandinsky: Über das Geistige in der Kunst insbesondere in der Malerei, Vor. u. Komment. zur rev. Neuaufl. v. Jelena Hahl-Fontaine, Einf. v. Max Bill, Bern (Benteli) 2004, S. 114. 


\section{HEINZ BRÜGGEMANN}

Die historische Tendenz dieser Überlegungen liegt in dem angedeuteten Versuch, die malerische Sprache als ein Medium zu verstehen, in dem auch die Sprachen der Komposition zu ihren Stimmen finden - "Farbensprache« hatte Kandinsky im ersten Entwurf von 1910 als Untertitel für Über das Geistige in der Kunst vorgesehen. Die briefliche Andeutung Benjamins, dass er in seinen Notizen "das Problem der Malerei in das große Gebiet der Sprache einmünden lasse, dessen Umfang" er »schon in der Spracharbeit andeute" (GB I, 395), führt hier weiter. In seinem Aufsatz Über die Sprache überhaupt und über die Sprache des Menschen betrachtet er als denkbar, dass die Sprache der Malerei in "gewissen Arten von Dingsprachen fundiert" sei, dass es sich um "namenlose, unakustische Sprache(n), um Sprache(n) aus dem Material« handle (GS II, 156). Die Auseinandersetzung zwischen Zeichen und Mal wirkt bei Benjamin fort in jener zwischen Form und Farbe, zwischen dem Linearen und dem Farbigen.

Es ist die Entgegensetzung des Linearen und der Farbigkeit als Grenzunbestimmten, die Benjamins sinnlich-mimetische Bestimmung des Farbensehens als Urphänomen der Phantasieanschauung aufgreift. In Wiederaufnahme der alten Diskussion über das Verhältnis von Umriss und Farbe, von disegno e colore, doch nun mit einer wahrnehmungstheoretischen, psycho-physischen Wendung: "Aller Form nämlich", heißt es, "allem Umriß, den der Mensch wahrnimmt, entspricht er selbst in dem Vermögen, ihn hervorzubringen. Der Körper selbst im Tanz, die Hand im Zeichnen bildet ihn nach und eignet ihn sich an. Dieses Vermögen aber hat an der Welt der Farbe seine Grenzen; der Menschenkörper kann die Farbe nicht erzeugen." (GS IV, 613) Darum, und hier erhält das Denken in Analogien wieder das Wort, »entspricht [er] ihr nicht schöpferisch, sondern empfangend: im farbig schimmernden Auge«. (Ebd.) Solcher Körper-Lektüre des Farbensehens ist Benjamin, wie fragmentarisch gebliebene Anmerkungen zeigen, auch beim »Erröten in Zorn und Scham» (GS VI, 120) nachgegangen. Das "Mal« führt er dort als "die Fläche» ein, "auf der von innen und außen her etwas zur Erscheinung kommen kann. Die Mauer. Das menschliche Antlitz«. (Ebd.) Während Benjamin mit dem menschlichen Antlitz in verschobener Form auf die farbtheoretische Diskussion über das Inkarnat, die Färbung der menschlichen Haut, anspielt, greift er mit dem Bild der »Mauer« zur Veranschaulichung des energetischen Unruhepotentials der Farbe als einem Aktanten geradezu einen Topos in der Diskussion über Farbe in Wahrnehmungspsychologie und Gestalttheorie um 1900 auf, der auf Leonardos Inspirationslehre zurückgeht. Die Mauer mit ihren Flecken und 


\section{WALTER BENJAMIN UND GERSHOM SCHOLEM}

durch Witterung hervorgebrachten Farben erscheint als Medium des aktiven und übergehenden Kolorits und zugleich als Stimulans gestaltender und entstaltender Phantasie. ${ }^{19}$

Benjamin hat die Konstellation von Zeichen und Mal immer wieder aufgegriffen, in verwandten Zusammenhängen bedacht, so in der Konfrontation von Malerei und Graphik, auf die er in einer Variante zur Zweiten Fassung des Essays Das Kunstwerk im Zeitalter seiner technischen Reproduzierbarkeit zu sprechen kommt. Sie zeigt in aller Deutlichkeit, wie er die von Scholem kantianisch gesetzte Antinomie mathematisch motivierter Raumzerlegung als primärer und Farbe als sekundärer Qualität unterläuft. In der Weise nämlich, dass er, vergleichbar (wenn auch nicht in forschungspraktischer Hinsicht) Aby Warburgs Verfahren im Vortrag über das Schlangenritual, historisch-anthropologisch, religions- und kulturwissenschaftlich, kunst- und wahrnehmungstheoretisch auf die Zeit vor dem Bild, auf vormoderne, ja vorzeitliche Funktionen von Linie und Farbe zurückgeht. Wenn er versucht, "Graphik und Malerei unterm Begriff des Kultwerts genau (zu) bestimmen « (GS VII.2, 676), wendet er sich von Kant zu Goethes zart-empirischer Erkenntnishaltung. Er fragt "nach den Korrespondenzen, die Graphik und Malerei in der Magie besitzen« und trägt diese Frage vor als die "nach den magischen Urphänomenen, die, in der Graphik einerseits, in der Malerei andererseits etwa beschlossen liegen« (ebd.). Dies explizit, um zum einen »die sinnlichen Differenzen zwischen Graphik und Malerei in der elementarsten Form zu erfassen«, zum andern dort, wo sie sich "am menschlichen Leib darstellen«, an der zentralen Instanz »des Magischen« (ebd.). Die Frage führt, was die Linie betrifft, auf den »Bannkreis«, in dem »der Kultwert der Linie sein Maximum» (ebd.) erreiche. Was die Farbe, das "Mal« angeht, gleicht die Antwort eher einer unabgeschlossenen Suchbewegung - außer vom Erröten als vorübergehender Färbung ist die Rede nun auch von gestaltähnlichen Lichtreflexen auf der Wand (den "farbigen Kringeln« aus einem Gedicht Chamissos), von der Wand im Palast des Nebukadnezar usf., um schließlich in die allgemeinste Bestimmung des malerischen Phänomens als Kultwert, des »Mals« zu münden: "eine farbige Konfiguration die auf der Wand erscheint (aus ihr heraustritt oder auf sie geworfen wird) - eine Konfiguration, die magisch angesehen, transitorisch, profan angesehen eher transportabel zu nennen wäre.« (GS VII.2, 677)

Welche künstlerischen Phänomene mit dieser allgemeinsten Bestimmung, die zudem in der Diagnose einer Krise der Malerei, ja in der Erwartung einer »Verkümmerung des Mediums der Malerei« (ebd.) fundiert ist, in den Blick genommen werden, kann man anhand

19 Vgl. dazu die instruktive Studie von Sabine Schneider: Verheißung der Bilder. Das andere Medium in der Literatur um 1900, Tübingen (Niemeyer) 2006, S. 46ff. und passim. Neben Helmholtz und Mach kann Schneider auch auf eine Reihe von Beispielen bei Meier-Graefe, Hugo von Hofmannsthal, Rilke u.a. verweisen. 


\section{HEINZ BRÜGGEMANN}

der letzten fragmentarischen Notaten zum Kunstwerk - Aufsatz nur vermuten. Eine Spur legen in dieser Hinsicht die varianten Passagen zur 1. Fassung, die sich mit der singulären Gestalt des Marcel Duchamps und der Herstellung surrealistischer Objekte befassen. Die spezifische Wirkung des Kunstwerks, so referiert Benjamin Duchamps, könne der heutige Mensch "weit eher an [zufälligen Konfigurationen im Abfall oder Schutt, an Gegenständen -] degagierten (das heißt ihrem Funktionszusammenhang entrückten Objekten (einer Klaviertasten tragenden Zimmerpalme, einem vielfach durchlochten Zylinderhut) als an beglaub[ig]ten Werken der Kunst erfahren«. (GS I.3, 1046) Benjamin attestiert dieser Form der Produktion, der »Herstellung sürrealistischer Objekte« - längst eine passionierende Beschäftigung für viele Maler aus diesem Kreis -, dass in ihr dem Zufall »ein großer Spielraum gewährt wird « (ebd.). So kommt mit dem Begriff des Spiels der Gegenpol des schönen (geschlossen, vollendeten) Scheins zum Tragen - er ist mit den aleatorisch motivierten artistischen Versuchsbildungen aufs engste verbunden. Benjamin stellt sie, denen er zumindest einen diagnostischen Wert zubilligt, nun aber in eine Tradition, die die Surrealisten selber schon für diese Hervorbringungen bemüht haben. In seinem Falle führt sie zurück zu jenem magischen Urphänomen, das in der Malerei beschlossen liegt, der aus der Wand heraustretenden farbigen Konfiguration. Man möge sich, notiert er, angesichts solcher spielerischen Produktionen

einer berühmten Stelle bei Lionardo erinnern. Lionardo berichtet, wie er gelegentlich seinen Schülern, die um Modelle verlegen gewesen seien, eine feuchte Mauer in ihrem Blickfeld mit den Worten gewiesen habe: diese möchten sie zum Modell nehmen; daraus würden sie alles lesen können, woran sie Bedarf hätten: Schlachten, Frauenleiber und Tiere. (Ebd.)

So führt die konstruierend aufs Älteste zurückgreifende Frage nach der Korrespondenz, die die Malerei in der Magie besitzt, zur hellsichtigen Diagnose jener zeitgenössischen Tendenzen der Avantgarde, die sich anschickt, nicht nur das Tafelbild, sondern auch den überlieferten Begriff der Kunst zu überwinden, aufzulösen. Damit ist zugleich jene Sphäre avisiert, die an anderer Stelle (s.o.) als die des noch gar nicht Vorstellbaren umschrieben wurde.

Eine ganz andere Perspektive auf den Kubismus (wie auch auf den Futurismus) gewinnt Benjamin ebenfalls im Zusammenhang des Kunstwerk-Aufsatzes - eine Perspektive, aus der sich nun auch die Antinomie von Zerlegung des Raums und Mitteilung verwandelt darstellt. Der Kubismus erscheint als ein Versuch der Kunst, der Art und Weise, in der im Film die Apparatur in die Wirklichkeit eingedrungen sei, Rechnung zu tragen - ein Prozess, den Benjamin mit dem Verfahren des Chirurgen, operativ in das Innerste des Behandelten zu 
dringen, verglichen hat (vgl. GS I, 495f. und 503). Scholems Bestimmung des Kubismus war: das Wesen des Raums, der die Welt ist, durch Zerlegung mitzuteilen. Kahnweilers kantianisch inspirierte Darstellung exponiert den wahrnehmungsgeschichtlichen Bruch weiter: mit der peinture conceptuelle sei die Wahrnehmungswelt in den Menschen selber versetzt. Beide Positionen zusammen genommen, lassen die Nähe zu Benjamins »Hilfskonstruktion«, dem »Begriff des Operateurs" (GS I, 495) sogleich deutlich werden. Der Operateur steht wie der Chirurg (in Entgegensetzung zum Magier) für den Kameramann, der (im Gegensatz zum Maler) »tief ins Gewebe der Gegebenheit« (GS I, 496) eindringt und ein »vielfältig zerstückeltes« Bild davonträgt, »dessen Teile sich nach einem neuen Gesetz zusammen finden«. (Ebd.) Dieses operative Eindringen ins opake Gewebe der gegebenen Wirklichkeit hat »in der ganzen Breite der optischen Merkwelt « eine »Vertiefung der Apperzeption zur Folge gehabt« (GS I, 498), die Benjamin in ihrer Wirkung der von Freuds Studie zur Psychopathologie des Alltagslebens vergleicht. So wie diese »Dinge isoliert und zugleich analysierbar gemacht « habe, "die vordem unbemerkt im breiten Strom des [scil. akustisch H.B.] Wahrgenommenen mitschwammen« (ebd.), so seien die "Leistungen, die der Film vorführt, viel exakter und unter viel zahlreicheren Gesichtspunkten analysierbar« als jene, "die auf dem Gemälde oder auf der Szene sich darstellen« (GS I, 498f.). Bis zu diesem Punkt handelt es sich lediglich um einen Vergleich der Verfahren, den Benjamin in der eher allgemein und neutral gefassten Behauptung gipfeln lässt, der Film habe die Tendenz, »die gegenseitige Durchdringung von Kunst und Wissenschaft zu befördern« (GS I, 499). An keiner Stelle wird für den Begriff eines Optisch-Unbewussten Freuds spezifische psychologische Ätiologie für die isolierten Dinge resp. Worte in Anspruch genommen. Erst dann, wenn Benjamin die spezifischen, bis dahin vor allem von Béla Balázs und Rudolf Arnheim analysierten filmischen Verfahren vorführt: die Großaufnahme mit ihrer Betonung versteckter Details an den uns geläufigen Requisiten, die Erforschung banaler Milieus "unter der genialen [sic! H.B.] Führung des Objektivs« (ebd.). Merkwürdig, dass hier ein Adjektiv aus einer doch historisch überwundenen Kunstlehre gebraucht wird!) etc. - erst dann findet sich eine Wendung, die dem analytischen Anspruch Freuds wenigstens nahe kommt. Der Film habe "auf der einen Seite die Einsicht in die Zwangsläufigkeiten vermehrt, von denen unser Dasein regiert wird“ (ebd.). Das wird an anderer Stelle durch das Motiv des mitspielenden Requisits illustriert: es zeige, wie uns die Materie mitspiele. Ob die so vermittelten Beobachtungen einen Vergleich mit Freuds Analyse des Triebhaft-Unbewussten in den Fehlleistungen etc. aushalten, muss man eher bezweifeln, allenfalls die zerstreute Aufmerksamkeit des Rezipienten als Bedingung der Möglichkeit solcher Einsichten scheint als vergleichbare Größe gegeben. 
Die andere Möglichkeit des Films bringt uns in größere Nähe zur gleichzeitigen bildenden Kunst - die nämlich, »eines ungeheuren und ungeahnten Spielraums uns zu versichern!« (GS I, 499). Die uns einschließende, undurchsichtige, weil auch durch Gewohnheiten befestigte alltägliche Umwelt - diese »Kerkerwelt«, wie Benjamin sie nennt, vermag der Film "mit dem Dynamit der Zehntelsekunden« (ebd.) zu sprengen. Und wir, die Zuschauer, können nun zwischen »ihren weitverstreuten Trümmern gelassen abenteuerliche Reisen unternehmen." (GS I, 500) Wenn die Haltung des Zuschauers als gelassen charakterisiert wird, dann entspricht das jener frühen Bestimmung der Phantasie als reiner Empfänglichkeit (s.o. S. 13), die sich vor allem gegenüber dem Aktiven und Übergehenden, dem Verflüssigenden und Überfliegenden der Farbe bewährt. Schon die haptische Form der Farbe impliziert Aufhebung von Distanz, die der Kamera überbietet sie noch, zumal alle Wahrnehmungsbedingungen des filmischen Raums auf Immersion angelegt sind. Die Kamera, so Béla Balázs, "nimmt mein Auge mit. Mitten ins Bild hinein. Ich sehe die Dinge aus dem Raum des Films. Ich bin umzingelt von den Gestalten des Films und verwickelt in seine Handlung, die ich von allen Seiten sehe. ${ }^{20}$ Balázs hebt auch sogleich das so zuvor in einer Kunst noch nicht gegebene Dispositiv zur Identifizierung hervor - ein Moment, das Benjamin auszublenden scheint, hier jedoch eher, um das Moment reiner Empfänglichkeit auch am Film darzustellen. Kennen die frühen Texte das in die Farbe zerstreute Wahrnehmen der Kinder, so entwickelt noch der Kunstwerk-Aufsatz, analog formuliert, das in die filmisch produzierten Wirklichkeits-Trümmer zerstreute Wahrnehmen der Kinozuschauer.

Benjamins Konstruktion des gelassen zwischen Trümmern Reisenden ist noch überwiegend von seiner kleinen Studie über Karl Bloßfeldts fotografische Mehrfach-Vergrößerungen von Pflanzen bestimmt, die "völlig neue Strukturbildungen der Materie» (GS I, 500), eine andere, apparaterzeugte Natur sichtbar werden ließen; er überträgt dieses Modell auf den Film am Beispiel der Zeitlupe, die in den bekannten Bewegungen ganz unbekannte entdecke - er beschreibt sie mit Rudolf Arnheim: »die gar nicht als Verlangsamungen schneller Bewegungen, sondern als eigentümlich gleitende, schwebende, überirdische wirken« (ebd.).

An dieser Stelle sind nun Zusammenhänge darzustellen und zu bedenken, die der Aufmerksamkeit der Forschung zum Phänomen des Optisch-Unbewussten bislang entgangen sind - wohl nicht zuletzt auch durch die Fixierung auf den Freud-Vergleich. Es existiert ein sog. Marseiller Entwurf von Siegfried Kracauers Theorie des Films, der erst 2005 durch den von Inka Mülder-Bach und Sabine Biebl betreuten 3. Band der Werkausgabe einem breite-

20 Béla Balázs: Der Geist des Films, Einleitung von Hartmut Bitomsky, Frankfurt a.M. (Makol-Verlag) 1972, S. 9/10 der Faksimile-Ausgabe Halle 1930, die diese Ausgabe wiedergibt. 
ren Publikum bekannt gemacht wurde. Dieser "Marseiller Entwurf « zu einer Theorie des Films erhalte, wie es in Nachbemerkung und editorischer Notiz heißt, »seinen explosiven Charakter" vor allem aus der »Engführung von historischem Schrecken und Medienreflexion« ${ }^{21}$ Bedeutsam wird er aber auch durch den Umstand, dass der im August 1940 aus Lourdes nach Marseille gekommene Walter Benjamin bis zu seinem Fluchtversuch Ende September "fast täglich mit Kracauer" zusammentraf ${ }^{22}$ und an der Entstehung dieses Entwurfs sicher mehr als nur Anteil nahm. Denn sieht man näher, scheint der Eindruck unabweislich, dass einige der Kategorien aus den filmtheoretischen Passagen des Kunstwerk-Aufsatzes von den Freunden noch einmal erörtert wurden - Gespräche, die ihre Spur in den Marseiller Heften hinterlassen haben. Das gilt, neben anderen (etwa dem Schluss vom Mitgespieltwerden durch die Materie auf Nähe zum Materialismus), sicher auch für Benjamins Kategorie des Optisch-Unbewussten. Mehrfach kommt der Entwurf beschreibend, dokumentierend, auf dieses Phänomen zurück, ohne es selber explizit so zu nennen.

Die Notate setzen ein mit dem von der Fotografie, zumal der Momentaufnahme, ausgehenden Gedanken, dass so wie sie »innerhalb der materiellen Komplexe nicht oder nur ungenau wahrgenommene Seinsbestände ${ }^{23}$ erschließt, der Film auch in der Totale »materielle Phänomene sichtbar machen« kann, »die sich der unmittelbaren Wahrnehmung entziehen, bzw. ungenügend apperzipiert werden« $(561) .^{24}$

Kracauer exponiert zunächst die konventionellen Bahnen, und das heißt zugleich die grundsätzliche Eingeschränktheit der menschlichen Wahrnehmung, um zu der entscheidenden These zu gelangen: so gewiss wie »der Film die ganze Welt ins Spiel zu mischen hat, so gewiß ist er dann rein bei sich selber, wenn er die materielle Dimension unter Ausschluß der intentionalen, d.h. ohne an irgendeinen Sinn zu rühren, durchmißt« (591). Unter einer Überschrift, die Benjamins Optisch-Unbewusstem sehr nahe kommt: „Die Phänomene, die nicht ins Bewußtsein zu treten pflegen« (599), führt er nach Maßgabe einer Psychologie und Phänomenologie der Wahrnehmung jene "Seinsbestände« auf, "die aus der konventionellen Wahrnehmung ausfallen«, wobei er hervorhebt, »dass es sich um Phänomene der Umgebung des Wahrnehmenden handelt, [...] die ihm zugänglich sein

21 Siegfried Kracauer: Theorie des Films. Die Errettung der äußeren Wirklichkeit. Mit einem Anhang "Marseiller Entwurf « zu einer Theorie des Films, hg. v. Inka Mülder-Bach, unter Mitarbeit von Sabine Biebl, Frankfurt a.M. (Suhrkamp) 2005, 3. Bd., S. 851.

22 Ebd.

23 Ebd., S. 565. Im Folgenden nach dieser Ausgabe mit Seitenzahl in Klammern zitiert.

24 In einer gestrichenen Formulierung heißt es dazu: der Mensch der Totale sei nicht darauf eingestellt, "die materiellen Phänomene als solche zu erfassen [...] Er nimmt sie unbewußt hin, aber nicht bewußt mit; er setzt sie vag voraus, aber nicht ihrer Eigentümlichkeit nach ein; er strebt mit seinen Intentionen über sie weg, statt sie selber zu intendieren.« (Ebd., S. 592) Das wäre die knappste Umschreibung des sog. OptischUnbewussten. 


\section{HEINZ BRÜGGEMANN}

könnten« (ebd.). Zu solchen »Ausfallerscheinung(en)« (ebd.) zählen: das Flüchtige, schnelle Bewegungen, das Spiel der Lichter und Schatten, sodann der Abfall, Müll, Schmutzwinkel, "alles das, was man hinter sich läßt, wovon das Leben sich abkehrt (so das Straßenpflaster)«(ebd.); am wichtigsten ist »der Ausfall des Gewohnten. Das was dem Menschen so gewohnt ist, dass es mit ihm verwachsen scheint, entzieht sich der Wahrnehmung« (ebd.). Zu dem »intim Gewohnten gehören: die Menschen des täglichen Umkreises [...]; die Kleider und das ganze Drum und Dran [...], die vertrauten Interieurs; die vertrauten Straßen und Wege usw.« (599, 601). Man kann diese Aufzählung wie eine detaillierte Explikation jener von Benjamin erwähnten Kerkerwelt des Alltäglichen lesen, die der Film aufsprengt und uns zugleich eines Spielraums in ihren Trümmern versichert. Kracauer beschreibt das Gewohnte als Begleiter des Menschen, es hafte »ihm wie eine Schleppe an, zu der auch das Flüchtige, der Abfall" gehöre: »Es ist ein Hautphänomen.« (601)

All diese Ausfallerscheinungen aber und vor allem das Gewohnte zu seinem Gegenstand zu erheben, ist die spezifische Leistung des Films - er vermag diese mit uns verwachsenen Phänomene »ihrem Ansich nach darzubieten, wenn sie plötzlich von uns abgetrennt erscheinen« (ebd.). Das kommt Benjamins Beschreibung des filmischen Verfahrens der Isolation von bisher unbemerkt im breiten Strom der Wahrnehmung mitschwimmender Phänomene, die im Freud-Vergleich terminiert, sehr nahe.) Kracauer nennt als filmische Verfahren die konventionelle Totale alter Filme, die uns die »Seinsbestände, mit denen wir damals zusammen gelebt haben - ohne eine Ahnung von ihrer intimen Zugehörigkeit zu uns", nun gleichsam nackt zeigen: "In diesem Mobiliar haben wir gehaust, gelitten? Es ist, als befänden wir uns plötzlich verwesten Teilen unseres gelebten Lebens gegenüber.« (603) Er nennt die Großaufnahmen, die Wahl der Einstellungen, die uns »das Intime, Vertraute« (ebd.) entfremden, nicht anders - so seine Korrespondenz zur gleichzeitigen bildenden Kunst - versuche der durch Film und Fotografie erst möglich gewordene Surrealismus "das Ansich des materiellen Zubehörs zu vermitteln, das uns umgibt und begleitet.« (ebd.; vgl. dazu oben die Ausführungen Benjamins zum surrealistischen Objekt). Er nennt schließlich die »Phänomene, die das Bewußtsein zertrümmern«, all jene »materiellen Phänomene, bzw. Vorgänge, die dem Menschen eine materielle Sensation verschaffen, ihm einen Schock versetzen« (ebd.). Für Kracauer setzt der Film, wenn er diese Schocks zum Gegenstand erhebt, nur »Traditionen fort, in denen sich das primitive Verlangen der Menschen, in die Tiefen des Körperlichen einzukehren und Schocks zu erleiden oder zu versetzen, seit alters her ausleben kann.« $(605)^{25}$

25 Ich habe hier (in knapper Form) allein auf den Text des Marseiller Entwurfs abgehoben, der zeitlich unmittelbaren Nähe zum Denken Benjamins halber. Es bleibt hinzuweisen auf die endgültigen Formulierungen 
Wenn er den Phänomen des Schocks zuschreibt, "rein auf die körperlich-materielle Konstitution, sie erschütternd und durcheinanderwirbelnd « (ebd.) zu wirken, dann argumentiert er eher von inhaltlichen Elementen des Films, von in ihm aufgehobenen Volkstraditionen her (Jahrmarkt, Grand Guignol); Benjamin hingegen verankert die Schockwirkung im filmischen Verfahren selber. Seine Darstellung der Kamera und ihrer Möglichkeiten ist nicht allein auf die von Arnheim exponierten schwebenden Aussichten beschränkt, er spricht von »ihrem Stürzen und Steigen, ihrem Unterbrechen und Isolieren, ihrem Dehnen und Raffen des Ablaufs, ihrem Vergrößern und ihrem Verkleinern« (GS I, 500).

Und vorzüglich aus dieser Perspektive kommen die Korrespondenzen zur zeitgenössischen bildenden Kunst, zum Dadaismus und Surrealismus zumal in den Blick. Benjamin hat sie als die Frage nach dem Verhältnis von Technik und Kunst zu systematisieren versucht. Jede ausgebildete Kunstform stehe im Schnittpunkt dreier Entwicklungslinien: 1. arbeite die Technik auf eine bestimmte Kunstform hin, 2. arbeiteten »die überkommenen Kunstformen in gewissen Stadien ihrer Entwicklung angestrengt auf Effekte hin, welche später zwanglos von der neuen Kunstform erzielt werden« (GS VII, 378), 3. arbeiteten »oft unscheinbare, gesellschaftliche Veränderungen auf eine Veränderung der Rezeption hin, die erst der neuen Kunstform zugute« (ebd.) komme (etwa die Panoramen auf das Kino).

Für den unter 2.) avisierten Vorgang nennt Benjamin den Dadaismus, er hat das Theorem in der XIV. und XV. These der dritten Fassung des Kunstwerk-Aufsatzes ausgeführt. Damit aber kommt eine Wirkung des Films zur Sprache, die den an Phantasie als reiner visueller Empfänglichkeit orientierten Darstellungen im Kontext von Vergrößerung und Zeitlupe, als deren haptische Form zur Seite gestellt werden müssen. Das Kunstwerk wurde »bei den Dadaisten zu einem Geschoß« (GS I, 502), begründet in der grundsätzlichen Entwürdigung seines Materials, im Abfall der Sprache ebenso wie in der Zerstörung der Aura durch die unstilisierte, unmittelbare bildnerische Verwendung von Objekten aus der alltäglichen Umwelt. Vergleichbar wird das dadaistische Artefakt dem Film durch seine »taktile Qualität« (GS I, 502) - eine von Alois Riegl entlehnte Kategorie, die die

in der veröffentlichten Fassung, die sich im Kapitel "Gewöhnlich unsichtbare Dinge« (ebd. S. 92-112) finden. Dieses Kapitel und die dazugehörigen Entwurfsskizzen eröffnen gänzlich neue Perspektiven für die Erforschung der Kategorie des Optisch-Unbewussten bei Benjamin, vor allem können sie die strikt wahrnehmungstheoretische Problemstellung wieder zur Geltung bringen. Einen ersten Versuch, die Kategorie des Optisch-Unbewussten im fotografie- und filmgeschichtlichen Zusammenhang darzustellen, bietet die Untersuchung von Andreas Becker: Perspektiven einer anderen Natur. Zur Geschichte und Theorie der filmischen Zeitraffung und Zeitdehnung, Bielefeld (Transcript) 2004 (sie geht freilich auf die hier für Kracauer und Benjamin dargestellten Korrespondenzen nur kursorisch ein). 


\section{HEINZ BRÜGGEMANN}

haptische Qualität des Bildes meint. ${ }^{26}$ Benjamin expliziert: „Es stieß dem Betrachter zu.» (ebd.) und er erläutert die Entsprechung zum taktilen Element des Films damit, dass dies »auf dem Wechsel der Schauplätze und Einstellungen beruht, welche stoßweise auf den Beschauer eindringen. (ebd.) Hier ist freilich nicht bedacht, dass eine relativ schnell eingetretene Habitualisierung der filmischen Wahrnehmung dieses Moment gleichsam abgeschliffen, entfärbt hat, während die provokative Kraft des Dadaismus in der musealen Präsentation zur Ruhe gekommen, damit zumindest in ihrer Potenzialität für jeden Betrachter konserviert, aufbewahrt ist.

Von dieser Wirkungs-Konstellation des Films mit dem Dadaismus her (die Kracauers Überlegungen zum Surrealismus entspricht) nimmt Benjamin nun den Kubismus und den Futurismus in den Blick. "Wie für den Dadaismus«, schreibt er, »sind dem Film auch für den Kubismus und Futurismus wichtige Aufschlüsse abzugewinnen« (GS I, 503). Benjamin konstruiert aus Logik und Teleologie eine Entwicklung, deren Maßstab die neue Kunstform des Films ist. Er vollzieht damit zu Teilen nur nach, was in der theoretischen Selbstreflexion der zeitgenössischen bildenden Kunst selber Thema war. Kracauer hat sich in jenem Abschnitt seiner Theorie des Films, der der Welt des Unbelebten gewidmet ist, nach der Berechtigung gefragt, diese Welt als einen besonderen filmischen Gegenstand zu charakterisieren und er zitiert einen Maler, Fernand Léger, „der scharfsichtig betont, dass einzig der Film dazu befähigt sei, uns mit Hilfe von Großaufnahmen für die Möglichkeit empfänglich zu machen, die in einem Hut, einem Stuhl, einer Hand und einem Fuße beschlossen liegen«. Er erwähnt eine ähnliche Äußerung von Cohen-Séat: »Und ich? sagt das fallende Blatt. - Und wir? sagen die Orangenschalen, der Windstoß ... Ihnen verhilft der Film, vorsätzlich oder nicht, zur Sprache. W Wenn der Film die Freiheit nutze, »um das Unbelebte zum Träger der Handlung zu machen«, so folge "er damit nur seiner Neigung zur Aufdeckung alles physischen Seins. ${ }^{27}$ Benjamin hat früh die Sprache der Malerei, der Farbe, in "gewissen Arten von Dingsprachen fundiert" gesehen, in Sprachen "aus dem Material« (GS II, 156 s.o.) Erst der Film hat, wie es scheint, diesen namenlosen, unakustischen Sprachen zu einem ganz neuen Klang verholfen.

26 Vgl. Alois Riegl: Spätrömische Kunstindustrie, Wien (Österreichische Staatsdruckerei) 1927, S. 32. Dort hat Riegl zunächst den auch von Benjamin an anderer Stelle verwandten Begriff "taktisch" verwandt. In einer Anmerkung des Herausgebers des Neudrucks, Emil Reisch, heißt es: »In einer Erwiderung auf Besprechungen seiner Arbeit [...] hat Riegl zugegeben, dass der Terminus taktisch schlecht gewählt sei und durch den Terminus haptisch überall zu ersetzen wäre." (Ebd.)

27 Kracauer: Theorie des Films (Anm. 21), S. 90. Wenige Seiten später zitiert er eine Äußerung von Louis Aragon: "Das Kino hat uns in wenigen Jahren mehr über den Menschen gelehrt, als Jahrhunderte der Malerei es vermochten: flüchtige Gesichtsausdrücke, kaum glaubhaftes und dennoch wirkliches Gebaren, Anmut und Häßlichkeit." (Ebd., S. 101) Unbeschadet ihres Wahrheitsgehalts machen diese Sätze doch einen kunsttheoretischen Gestus deutlich, der dem Benjamins zumindest verwandt ist. 
Kracauers theoretischer Impuls, dem es um nicht weniger als die Errettung der äußeren Wirklichkeit (so bekanntlich der Untertitel seines Buches) zu tun ist, erkennt im Film das Medium, dem physischen Sein in bisher ungeahnter Weise nahe zu kommen, ja geradezu in ein Verhältnis sprachlichen Austauschs mit ihm zu treten. Dabei ist die Referenz des Mediums auf äußere Wirklichkeit fraglos vorausgesetzt. Das ist sie bei Benjamin auch, aber sein Ausgangspunkt ist die Technik selber, m.a.W. die mit der Entwicklung der Kamera gegebene Durchdringung der Wirklichkeit mit der Apparatur, die eine andere, gleichsam durch die Apparatur erzeugte Natur sichtbar macht. Dem Film sei es gelungen, die »Apparatur für die künstlerische Darstellung der Realität« (GS I, 503) zu verwerten. Kubismus und Futurismus erscheinen nun, vom Film her gesehen, "als mangelhafte Versuche der Kunst, ihrerseits der Durchdringung der Wirklichkeit mit der Apparatur Rechnung zu tragen« (ebd.). Statt die Apparatur für die künstlerische Darstellung der Realität zu verwerten - was im übrigen wohl nur um den Preis ihrer Selbstaufgabe als Malerei möglich gewesen wäre -, hätten sie ihren Versuch »durch eine Art von Legierung von dargestellter Wirklichkeit und dargestellter Apparatur« (ebd.) unternommen. Die vorwiegende Rolle im Kubismus habe dabei »die Vorahnung von der Konstruktion dieser Apparatur « gespielt, "die auf der Optik« beruhe, im Futurismus hingegen »die Vorahnung der Effekte dieser Apparatur, die im rapiden Ablauf des Filmbands zur Geltung kommen." (Ebd.)

In dieser Konstruktion ist die Metapher der Vorahnung deutlich einer konstruierten geschichtsteleologischen Entwicklungslogik geschuldet. Schon von den historischen Daten her kann im Blick auf das Verhältnis beider Kunstrichtungen zum Film kaum von einer "Vorahnung" gesprochen werden. Beide haben ihren Ursprung im Bruch mit der zentralperspektivischen Raumkonstruktion, im Versuch einer Überwindung der konventionell, im Gegenüber von Subjekt und Objekt, erstarrten Wahrnehmungswelt. In der Tat gibt es eine Affinität zur filmischen Apparatur insofern das Dargestellte nicht mehr als in Form distanzierter Gebilde zur naturalistischen Beobachtung vor Augen liegt, der Bild-Raum nicht allein optisch schematischer, mathematisch bestimmter, homogener und abstrakter ist, sondern der Mensch in seine Welt situiert erscheint: die Dinge kreisen in der Empfindung und der Mensch lebt und wirkt in ihrem Kern, wie es Carl Einstein formuliert hat. ${ }^{28}$ Der Kubismus sucht die Ansichtenmannigfaltigkeit bewegten Sehens, orientiert an der physiologischen Optik, in den Bild-Raum zu integrieren, gut zehn Jahre nach den ersten Präsentationen des Stummfilms; der Futurismus drängt mit der Programmatik lo spettatore nel centro del quadro, der Betrachter mitten ins Bild, auf eine dynamisch-

28 Vgl. dazu vom Verfasser: Architekturen des Augenblicks. Raum-Bilder und Bild-Räume einer urbanen Moderne in Literatur, Kunst und Architektur des 20. Jahrhunderts, Hannover (Offizin) 2002, S. 403. 


\section{HEINZ BRÜGGEMANN}

immersive Bildstruktur, die über die Grenzen des Tafelbildes hinaus drängt. Und wenn er dem Postulat: Öffnen wir die Figur und schließen die Umwelt in sie ein, konsequent folgt, findet er in der Tat im Film seine adäquaten künstlerischen Lösungsmöglichkeiten, wie die futuristische Fotografie und der Film zur gleichen Zeit dann auch vor Augen führt.

Benjamins geschichtsteleologische und entwicklungslogische Konstruktion verfährt ihrerseits nach der Maßgabe bildlichen Denkens. Sie operiert mit einer Übertragung: indem der Film mit seiner künstlerischen Verwertung der technisch möglich gewordenen Durchdringung von Apparatur und Wirklichkeit als Fluchtpunkt in dieser kunstgeschichtlichen Konstruktion fungiert, wird das ganze komplexe Bedingungsgeflecht, aus dem Kubismus und Futurismus hervorgehen, einer Engführung in Hinsicht auf die durch ihn gesetzten Fluchtlinien unterworfen - gleichsam more geometrico wie im Bildspender dieser Übertragung, in der monokularen zentralperspektivischen Raumkonstruktion selber auch. Nur so kommt auch der metaphysische Überhang in Kategorien wie »Vorahnung « zustande, sie entspringt dem Bilderdenken von Fluchtlinien und Fluchtpunkt selber. Man hat es aber mit überwiegend gleichzeitigen Bedingungszusammenhängen zu tun, in denen das Verhältnis der Kunst zur Wissenschaft etwa der Sinnesphysiologie, zur Eigenlogik der künstlerischen Raumkonzeption seit Cézanne etc. eine ähnlich große Rolle spielt wie der Bezug auf die voraufgegangene und aktuelle Medienentwicklung. Aus solcher Gleichzeitigkeit entstehen auch Phänomene wie jenes, dass der Film nun seinerseits Bild- und Formsprachen der aktuellen Kunstentwicklung aufgreift - so im futuristischen und expressionistischen Film.

Es sei noch einmal Benjamins Argument zum Kubismus in der Fußnote des Kunstwerk-Aufsatzes erinnert: er verwerte die Apparatur (i.e. die filmische) für die künstlerische Darstellung »durch eine Art von Legierung von dargestellter Wirklichkeit und dargestellter Apparatur", wobei er sich, sozusagen, im Aggregatzustand der "Konstruktion dieser Apparatur« bewegt, »die auf der Optik beruht" (GS I, 503). An diesem Argument ist die Behauptung am meisten diskussionsbedürftig, dass dargestellte Wirklichkeit und dargestellte Apparatur im kubistischen Bild-Raum allenfalls durch eine Legierung verbunden sein sollen. Sie erinnert deutlich an oben erörterte Positionen: z.B. die von der Logik wie Teig durchknetete Realität (Gehlen). Kahnweiler (Daniel Henry) ist dem von Benjamin Gemeinten vielleicht am nächsten, wenn er schreibt: der Gegenstand entstehe aus dem Raumkonstruktiven, er beherrsche es nicht mehr (s.o.). Diese Dominanz des Raumkonstruktiven, der stereometrischen Zeichnung in der Fläche, erscheint bei Benjamin als dargestellte Apparatur, sie erscheint ihm mit der dargestellten Wirklichkeit bloß legiert. Gewiss gibt es weniger gelungene Arbeiten, in denen das Raumkonstruktive den Bild-Raum und 
das Mitgeteilte (mit Benjamin zu sprechen) überwuchert, zudeckt, aber in den gelungenen Arbeiten ist die Durchdringung von Formsprache und `Mitgeteiltem` eben nicht aufzutrennen, sondern dass der Gegenstand als ganz aus dem Raumkonstruktiven entstanden vorgestellt wird, verleiht ihm zwar eine kristalline Geschlossenheit, in der aber zugleich die Ansichtenvielfalt bewegten Sehens bildnerisch realisiert ist und so dem Betrachter mit der Anmutung selber entdeckenden Sehens entgegentritt. Gerade die gleichsam reflektiert mitgemalten Bedingungen der Wahrnehmung selber (die dargestellte Wahrnehmungswelt) haben diesen Bildern die konzentrierte Welthaltigkeit poetischer, lyrischer Gebilde verliehen (vgl. etwa Mallarmé und zahlreiche kubistische lyrische Versuche), die mehr ist als eine lose Legierung von dargestellter Apparatur und dargestellter Wirklichkeit.

Die Entgegensetzungen aus der frühen Diskussion mit Scholem kehren hier verwandelt wieder. Benjamin hatte gegen Scholems Bestimmung des Kubismus: das Wesen des Raumes, der die Welt ist, durch Zerlegung mitzuteilen, auf dem Verhältnis der Malerei zu ihrem sinnlichen Gegenstand insistiert und am Beispiel des Bildes von Picasso gefordert, die Mitteilung müsse den Gegenstand, nämlich »Dame mit Fächer« betreffen. Wenn er nun die dargestellte Apparatur - das wäre Scholems emphatisch herausgestellte Affinität des Kubismus zur Mathematik auf konstruierte Optik und vorgeahnte Apparatur bezogen - mit der dargestellten Wirklichkeit - den sinnlichen Gegenstand dargestellter Wirklichkeit - allenfalls im Zustand einer Legierung, eines äußerlich gestifteten Zusammenhangs, sehen mag, dann ist darin eine Abspaltung vorausgesetzt, deren Aufhebung er von Scholem gefordert hatte.

Aus den Reflexionen zu Zeichen und Mal, den magischen Urphänomenen von Graphik und Malerei, aber kommt dem Film zugute, dass in seiner künstlerischen Verwertung der Durchdringung der Wirklichkeit mit der Apparatur ein Moment überwiegt, das Benjamin für die Malerei, die Farbe zumal stark gemacht hat: das Moment des Unverfügbaren, Zufälligen. Das ist (mit Kracauer zu reden) seine Fähigkeit, »die materielle Dimension unter Ausschluß der intentionalen, d.h. ohne an irgendeinen Sinn zu rühren« (s.o.), zu durchqueren. Aus den frühen Reflexionen zum Farbensehen, zu Zeichen und Mal, sind in die Theorie des Films neben dem Unverfügbaren eingegangen: die reine Empfänglichkeit als rezeptive, gelassene Haltung, zumal angesichts des Übergehenden und Überfliegenden; das vormals in die Farbe nun ins Filmbild selber zerstreute Wahrnehmen als Form der Immersion; die Zugehörigkeit des Films zum Spiel als offener ästhetischer Versuchsbildung für die rezeptiven Reisen des Zuschauers; die Fähigkeit des Films, der (unakustischen) Sprache der Dinge, dem Ansich der materiellen Phänomene (mit Kracauer), Stimme und belebte Gegenwart zu leihen, das Nicht-Sichtbare darstellbar 


\section{HEINZ BRÜGGEMANN}

zu machen. Von der reinen Farbe als Wolkenheimat, als Medium der Phantasie, spricht für den Film auf seine Weise Siegfried Kracauer, wenn er die Dinge, »die normalerweise nicht gesehen werden«, verhandelt: das flüchtig Vorübergleitende, das rasch Vergängliche - »ein über die Ebene dahinziehender Wolkenschatten, ein im Winde treibendes Blatt«. »Dergleichen wie Traumelemente entschwebende Eindrücke können den Kinobesucher noch lange in Bann halten, wenn die Story, die sie darstellen helfen, schon in Vergessenheit gesunken ist. ${ }^{29}$ All dies sind Elemente aus jener Sphäre des Films, die Kracauer das Erschließen der materiellen Dimension, Benjamin das Optisch-Unbewusste genannt hat.

29 Kracauer: Theorie des Films (Anm. 21), S. 101. Zitat im Zitat Alexandre Arnoux ebd. nachgewiesen. 